\title{
Intraventricular haemorrhage in pregnancy due to Moya-moya disease
}

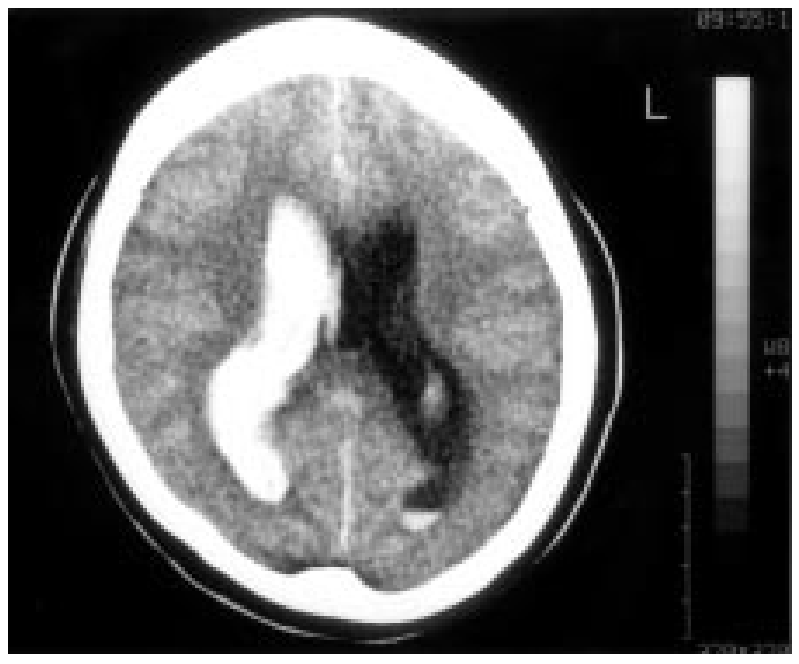

Figure 1 brain CT showing acute intraventricular haemorrhage.

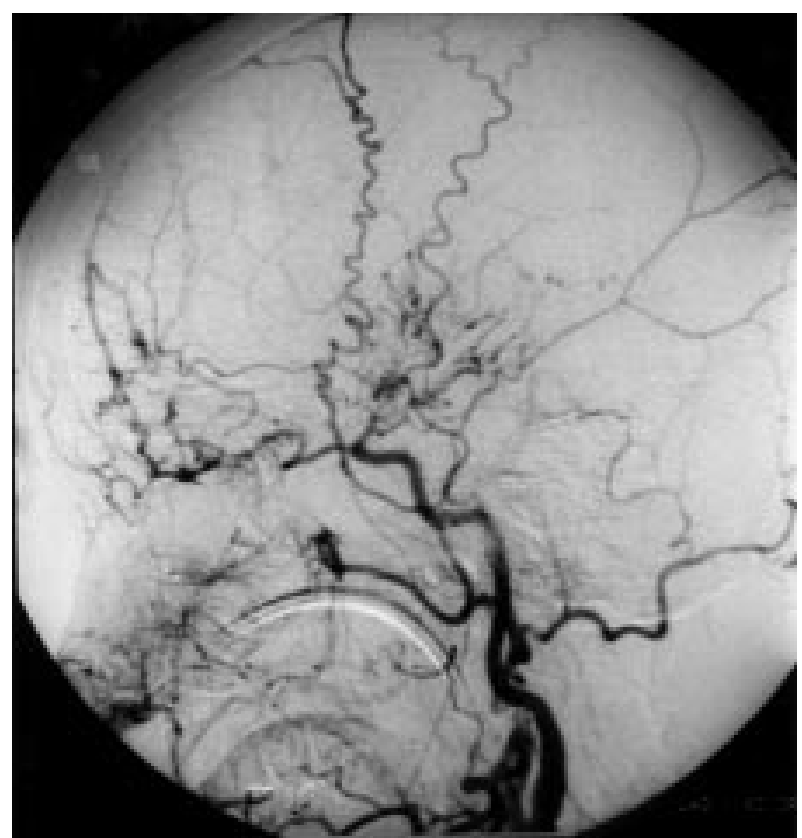

Figure 2 Left common carotid arteriogram, lateral view, showing occlusion of the supraclinoid portion of the internal carotid artery with filling of small branches of the middle cerebral artery via anastomoses from dural branches of the external carotid artery.

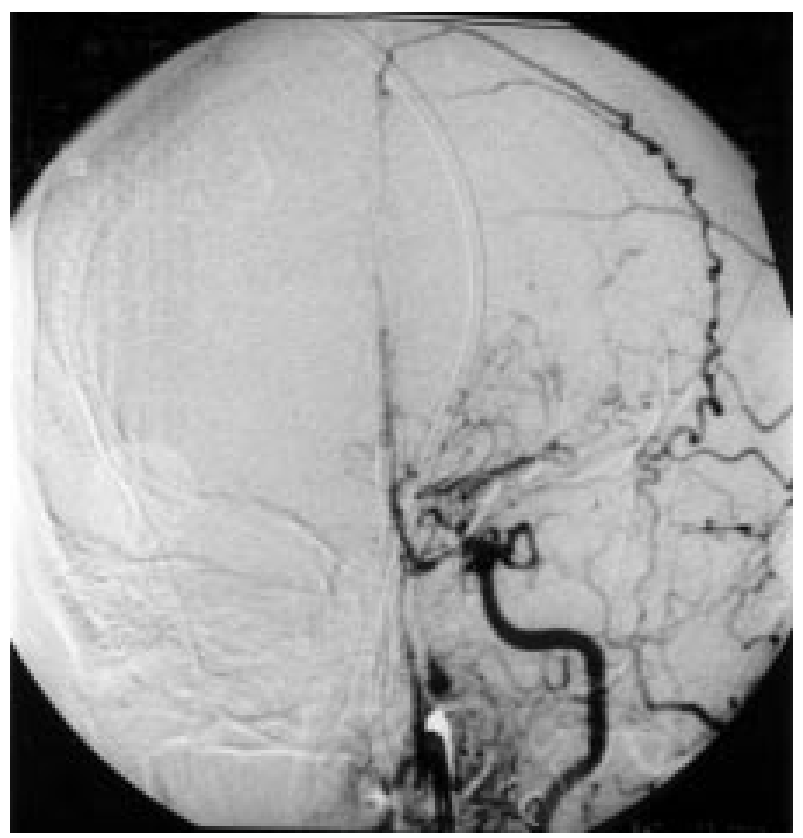

Figure 3 Left common carotid arteriogram, anterioposterior view showing filling of the anterior cerebral artery via anastomoses from branches of the external carotid artery.

A 37 year old Vietnamese woman 30 weeks into her first pregnancy presented with sudden severe headache and progression to unconsciousness over 30 minutes. In 1991 she was diagnosed as having migraine and a single generalised convulsion; an EEG and brain CT at that time were normal. There was no family history of note and she was taking no medication. On admission she was obtunded with pronounced neck stiffness and low grade pyrexia; there was no response to speech and pain produced incomprehensible sounds and limb withdrawal, there was no eye opening. Pupils were reactive to light and fundi were normal. Limb tone and tendon reflexes were generally increased with flexor plantar responses.

Brain CT (fig 1) showed extensive intraventricular haemorrhage with hydrocephalus. Cerebral angiography (figs 2, 3) disclosed typical appearances of Moya-moya disease, a diagnosis not previously made, with occlusion of the supraclinoid portion of both internal carotid arteries and the presence of abnormal collateral vessels. Abdominal ultrasound confirmed the presence of a viable fetus.

An external ventricular drain was inserted, and removed after three days. Over the course of 10 weeks she recovered with no major neurological sequelae but the headache persisted. Four weeks after admission she gave birth to a healthy baby by normal delivery.

PIERS NEWMAN ALI AL-MEMAR Department of Neurology, Queen Elizabeth Neurosciences Centre, Queen Elizabeth Hospital,
Birmingham, UK 